\title{
Provider Perspectives on Integrating Primary and Behavioral Health: A Report from the High Plains Research Network
}

\author{
Gregory Burfeind, MD, Deborah Seymour, PhD, Stefan H Sillau, PhD, \\ Linda Zittleman, MSPH, and John M. Westfall MD, MPH
}

Background: Integrated primary care, a health care delivery model that combines medical and behavioral health services, provides better patient access to care at a lower cost, with better outcomes compared with usual nonintegrated care models. The perspectives of primary care providers (PCPs) and behavioral health care providers (BHPs) toward integration are especially valuable because their input and endorsement are key to successful and sustained integration. However, there is little research assessing or comparing PCP and BHP perspectives on integration, especially in rural areas. The objective of this study was to identify rural PCP and BHP perspectives on integration.

Methods: Written and electronic surveys were distributed to PCPs and BHPs in the High Plains Research Network in rural eastern Colorado. Items included perspectives on improving behavioral health care, barriers to integration, and confidence in the ability to integrate primary and behavioral care.

Results: Surveys were completed by 88 PCPs (60\%), and 49 BHPs (63\%), for an overall response rate of $61 \%$. PCPs were significantly more likely than BHPs to prefer improving referral methods (odds ratio $[\mathrm{OR}], 2.2 ; P=.03)$ and significantly less likely to prefer colocation $(0 \mathrm{R}, 0.2 ; P<.0001)$, warm handoffs $(0 R, 0.3 ; P<.01)$, improved behavioral health training for PCPs $(0 R, 0.4 ; P<.01)$, and shared visits $(\mathrm{OR}, 0.4 ; P=.03)$ as ways to improve health care. Lack of sufficient methods of payment for behavioral health care services was the most commonly selected barrier to integration by both groups. PCPs were significantly more likely than BHPs to select recruitment $(0 R, 3.8 ; P<.001)$ and retention $(0 R, 2.7 ; P<.01)$ of behavioral health care staff as a major barrier. BHPs were slightly more optimistic than PCPs about the achievability of integration.

Conclusions: Important differences of perspective exist between rural PCPs and BHPs regarding the best ways to improve behavioral health care, barriers to integration, and the achievability of integration. These differences may have important implications for rural communities and health care systems considering a transition to an integrated primary care model. (J Am Board Fam Med 2014;27:375-382.)

Keywords: Integrated Health Care Systems, Mental Health, Primary Health Care, Rural Health

Most patients with mental health and substance use disorders (jointly referred to as behavioral health conditions) are initially evaluated and subsequently receive the majority of treatment for these conditions in

This article was externally peer reviewed.

Submitted 12 May 2013; revised 9 October 2013; accepted 11 October 2013.

From the High Plains Research Network, Department of Family Medicine, University of Colorado School of Medicine, Aurora.

Funding: none.

Conflict of interest: none declared.

Corresponding author: John M. Westfall, MD, MPH, University of Colorado, PO Box 6508, Aurora, CO 80045-0508 (E-mail: Jack.Westfall@ucdenver.edu). the primary care setting. ${ }^{1,2}$ Patients strongly prefer to receive behavioral health care within the primary care setting. ${ }^{3}$ In addition, up to $70 \%$ of all primary care visits are related to behavioral health needs, whether explicit or masked as somatic complaints. ${ }^{4}$ Taken together, it is not surprising that primary care is considered by many to be the de facto behavioral health system in the United States. ${ }^{5-7}$ Unfortunately, many common behavioral health disorders continue to be underdiagnosed and inadequately treated by primary care providers. ${ }^{8-10}$

Integrating behavioral health care into primary care (integrated primary care) is an effort to improve diagnosis and treatment of behavioral health condi- 
tions commonly seen in primary care. It "combines medical and behavioral health services to more fully address the spectrum of problems that patients bring to their primary medical care providers. It allows patients to feel that, for almost any problem, they have come to the right place." 11 The idea is to improve patient outcomes by encouraging collaboration between primary care physicians (PCPs) and behavioral health providers (BHPs) in the diagnosis and comanagement of behavioral health conditions. ${ }^{12}$

Studies have demonstrated that the clinical and functional outcomes of integrated primary care are superior to those of nonintegrated models of care for a variety of conditions, including depression, bipolar disorder, generalized anxiety disorder, panic disorder, and Alzheimer disease. ${ }^{3,12}$ These findings persist across a variety of age and ethnic groups, often with long-term improvements. ${ }^{3,12,13}$ Access to behavioral health care, engagement in treatment, and provider satisfaction also are improved. ${ }^{14-16} \mathrm{Fi}$ nally, integrated primary care has been shown to be more cost-effective than care as usual. ${ }^{12}$

Despite overwhelming evidence of the efficacy of integrated primary care, its successful and sustained implementation is difficult. A considerable amount of research has been devoted to integration barriers. By far the most consistently cited barriers are financial in nature. Some other commonly cited provider- and system-level barriers include reimbursement, staffing, and lack of a program champion to spearhead the integration effort. Nearly all key informants interviewed by Kathol et $\mathrm{al}^{1}$ indicated that the current reimbursement system is not amendable to sustaining integrated practice models because it segregates behavioral and physical health. Because of a shortage of licensed BHPs and limited financial resources, another common barrier is hiring BHPs. ${ }^{1,12,17}$ Finally, several researchers have identified the importance of a committed program champion and the lack thereof as a barrier. ${ }^{1}$

Implementation of integrated primary care in rural areas faces several additional challenges, most notably a higher prevalence of behavioral health conditions and difficulties with "accessibility, availability, and acceptability of mental health services." ${ }^{18}$ Rural youth and adults suffer from depression and substance abuse at higher rates than nonrural youth and adults, and they also have a higher rate of completed suicides. ${ }^{18,19}$ As of 1999 , $87 \%$ of mental health professional shortage areas are in nonmetropolitan areas, making access to be- havioral health care a major difficulty. ${ }^{17}$ The stigma surrounding mental health persists and is especially strong in rural areas; maintaining confidentiality is difficult, as in the classic example of recognizing a neighbor's vehicle parked in a mental health facility's parking lot. These factors work together to decrease the likelihood that rural residents will seek care for behavioral health problems. ${ }^{11,18}$

Regardless of how well designed an integrated primary care system is, both PCPs and BHPs must "buy in" for it to succeed and remain viable; therefore, obtaining the perspectives of these 2 groups is an important part of the integration process and can help identify philosophical and practical discrepancies between the 2 that may hinder successful integration. Proactively addressing these concerns has the potential to expedite the integration process and produce a more sustainable and successful end product. With additional challenges faced in rural areas, the importance of a well-informed and proactive approach to integration cannot be overemphasized. Despite this, little published research assessing or comparing PCP and $\mathrm{BHP}$ perspectives on integration exists, especially for rural providers. Based on the interest of the providers and community members in the High Plains Research Network (HPRN), we undertook this study is to identify rural PCP and BHP perspectives on improving behavioral health care, barriers to integration, and the achievability of integration in rural communities in the hope of providing a starting point for rural communities and health care systems interested in integrated primary care.

\section{Methods}

The HPRN is a practice-based research network encompassing all 16 counties of rural eastern Colorado, covering 30,000 square miles and 150,000 people. ${ }^{20}$ The HPRN includes 146 allopathic and osteopathic physicians, nurse practitioners, and physician assistants in 56 practices as well as 79 licensed clinical psychologists, professional counselors, psychiatrists, and social workers in 25 practices. More than $95 \%$ of the physicians in the HPRN are in family medicine. Two community mental health centers (CMHCs) have multiple sites of care (20 sites in 15 counties), none of which were colocated with a PCP at the time of the survey. Surveys from these sites were distributed and col- 
lected by the CMHCs and could not be distinguished based on geographic practice site. To our knowledge, only 4 primary care practices had a colocated, full-time BHP at the time of the survey. Two other practices have part-time BHPs (1 to 2 days per week). Anonymous written or electronic surveys were distributed to all providers in the HPRN during the summer of 2011.

Participants were asked whether the practice where they work uses an integrated BHP. To assess perspectives on improving behavioral health services in their communities, participants were asked to select up to 4 ideas from a list that included improving behavioral health training for PCPs; improving the behavioral health referral process; building an inpatient behavioral health facility; improving provider and patient access to BHPs via phone, E-mail, or videoconference; colocation of behavioral health and primary care; hiring a behavioral health case manager; using warm hand-offs; and implementing shared visits. We defined a warm hand-off as the opportunity to directly introduce the patient to the BHP at the time of the individual's medical visit, establishing an initial, personal contact between the patient and the behavioral health clinician. Participants also were asked to select the single best idea. To assess perspectives on barriers to integration in their practices and communities, participants were asked to select from a list that included lack of community support, no program champion, recruiting and retaining behavioral health staff, recruiting and retaining PCPs, lack of payment methods for behavioral health care, and limited resources and technical assistance for administrators and support staff. Participants also were asked to indicate the most important barrier. Space was provided for open-ended responses to both of these items.

Participants were asked to rate, using a 5-point Likert scale, the extent to which they agreed or disagreed with the statement, "Integrating behavioral health care and primary medical care in my community is an achievable goal." An open-ended question asking, "What is the ideal way to provide behavioral health services to people in your community?" was included. Finally, demographic and practice data were collected, including age, sex, race, years in practice, years in practice at current location, professional degree, and type of practice (private, public, for profit, not for profit, federally qualified health center, and rural health center).
The Colorado Multiple Institutional Review Board approved the study protocol and survey instruments.

Each idea for improving behavioral health care, barrier, practice, and race/ethnicity was considered separately as an indicator function. Achievability was scored on a 5-point Likert scale. Frequency tables were obtained for all categorical variables, and means tables were computed for continuous or scale variables for both the BHP and PCP groups. Categorical variables were compared between groups using 2-way tables and $\chi^{2}$ tests as well as Fisher exact association tests as needed. For binary outcomes, odds ratios (ORs) and confidence intervals (CIs) were calculated when possible. Continuous or scale variables were compared between groups using $t$ tests and Wilcoxon tests. Logistic regression was conducted to assess the effect of practice type (private, public) and provider characteristics (sex, race, years of practice, years at location) on the odds of agreeing with individual ideas for improvement and barriers, controlled for provider group. Because of sample size constraints, all models used only group and one other explanatory variable. Both interaction and additive models were considered. The interaction was tested and eliminated if it was not statistically significant. Standard regression models were fit for the achievability score. All calculations were performed using SAS software versions 9.2/9.3 (SAS, Inc., Cary, NC).

\section{Results}

Of 146 PCPs, 88 responded, and 49 of 78 BHPs responded, for response rates of $60 \%$ and $63 \%$, respectively, and a total response rate of $61 \%$. Among the practices, 44 of 56 primary care practices $(79 \%)$ and 13 of 25 behavioral health practices (52\%) were represented by survey respondents. Respondents' age, sex, race, and practice type are summarized in Table 1.

Table 2 shows that $24 \%$ of PCPs and $22 \%$ of BHPs currently work in a practice with an integrated BHP. Among BHPs, 24\% were working at a practice that was in the process of integrating a BHP, compared with only $2 \%$ of PCPs; $73 \%$ of PCPs did not work in a practice with an integrated BHP and were not considering adding one, compared with only $39 \%$ of BHPs.

Among PCPs, improving the referral process to the local CMHC was the most commonly selected 
Table 1. Demographics of High Plains Research Network Primary Care and Behavioral Health Provider Survey Respondents, 2011

\begin{tabular}{|c|c|c|}
\hline Demographics & $\begin{array}{c}\text { PCPs } \\
(\mathrm{n}=88)\end{array}$ & $\begin{array}{c}\text { BHPs } \\
(\mathrm{n}=49)\end{array}$ \\
\hline \multicolumn{3}{|l|}{ Age (years) } \\
\hline $25-44$ & $38(43)$ & $21(43)$ \\
\hline $45-64$ & $40(45)$ & $15(31)$ \\
\hline$\geq 65$ & $1(1)$ & $9(18)$ \\
\hline Refused & $9(10)$ & $4(8)$ \\
\hline \multicolumn{3}{|l|}{ Sex } \\
\hline Male & $38(43)$ & $14(29)$ \\
\hline Female & $40(45)$ & $33(67)$ \\
\hline Refused & $10(11)$ & $2(4)$ \\
\hline \multicolumn{3}{|l|}{ Race } \\
\hline White & $62(70)$ & $42(86)$ \\
\hline Black & $3(3)$ & $3(6)$ \\
\hline Asian & $8(9)$ & $0(0)$ \\
\hline Hispanic/Latino & $6(7)$ & $1(2)$ \\
\hline Other/refused & $10(11)$ & $3(6)$ \\
\hline \multicolumn{3}{|l|}{ Practice type* } \\
\hline Private & $24(27)$ & $13(27)$ \\
\hline Public & $8(9)$ & $0(0)$ \\
\hline For profit & $6(7)$ & $7(14)$ \\
\hline Not for profit & $8(9)$ & $9(18)$ \\
\hline FQHC & $19(22)$ & $2(4)$ \\
\hline $\mathrm{RHC}$ & $39(44)$ & $2(4)$ \\
\hline Other & $3(3)$ & $7(14)$ \\
\hline \multicolumn{3}{|c|}{ Provider training/education } \\
\hline MD/DO & $49(56)$ & - \\
\hline Physician assistant & $10(11)$ & - \\
\hline Nurse practitioner & $23(26)$ & - \\
\hline Psychiatrist & - & $1(2)$ \\
\hline $\mathrm{PhD} / \mathrm{PsyD}$ & - & $2(4)$ \\
\hline MA/MS & - & $19(39)$ \\
\hline LPC/PC & - & $10(20)$ \\
\hline MSW & - & $5(10)$ \\
\hline MFT & - & $5(10)$ \\
\hline Other/missing & $6(7)$ & $7(15)$ \\
\hline
\end{tabular}

*Providers could choose all that apply.

BHP, behavioral health provider; PCP, primary care provider; FQHC, federally qualified health center; RHC, rural health center; $\mathrm{MD}$, medical doctor; $\mathrm{DO}$, doctor of osteopathy; $\mathrm{PhD}$, doctor of philosophy; PsyD, doctor of psychology; LPC, licensed professional counselor; PC, professional counselor; MA, master of arts; MS, master of science; MSW, master of social work; MFT, marriage and family therapist.

idea for improving behavioral health services (66\%), whereas warm hand-offs was the most commonly selected idea among BHPs (65\%). Table 3 describes other commonly chosen ideas. PCPs chose improve access to BHPs via telephone, E-mail, or videoconference $(57 \%)$ and hire a case manager (42\%).
Table 2. Primary Care and Behavioral Health Integration of Current Practice Site

\begin{tabular}{lcc}
\hline Integration Status & $\begin{array}{c}\text { PCPs } \\
(\mathrm{n}=88)\end{array}$ & $\begin{array}{c}\text { BHPs } \\
(\mathrm{n}=49)\end{array}$ \\
\hline No integrated BHP in practice & $64(73)$ & $19(39)$ \\
Thinking about integrating a BHP & $1(1)$ & $6(12)$ \\
In the process of integrating a BHP & $2(2)$ & $12(24)$ \\
Integrated BHP & $21(24)$ & $11(22)$ \\
$\begin{array}{l}\text { Previously unsuccessful attempt to } \\
\text { integrate BHP }\end{array}$ & $1(1)$ & $0(0)$ \\
\hline
\end{tabular}

Data are $\mathrm{n}(\%)$.

$\mathrm{BHP}$, behavioral health provider; PCP, primary care provider.

Other commonly chosen ideas among BHPs were improve behavioral health training for PCPs (51\%), colocate $(51 \%)$, improving the referral process to the local CHMC (47\%), and improve PCP/ patient access to BHPs via telephone, E-mail, or videoconference (41\%).

Statistically significant differences in response rates existed between PCPs and BHPs for several ideas for improving behavioral health care. PCPs were significantly less likely than BHPs to select colocation (OR, 0.2; 95\% CI, 0.1-0.4), warm-hand offs (OR, 0.3; 95\% CI, 0.1-0.7), improve behavioral health training for PCPs (OR, 0.4; 95\% CI, $0.2-0.8$ ), and shared visits (OR, 0.4; 95\% CI, $0.2-$ $0.9)$. PCPs were significantly more likely than BHPs to select improving the referral process $(\mathrm{OR}$, 2.2; 95\% CI, 1.1-4.5).

Table 4 illustrates that lack of sufficient methods of payment for behavioral health care services was the most commonly selected barrier to integration by both PCPs and BHPs (61\% and 37\%, respectively). Other commonly selected answers among PCPs were recruitment and retention of appropriately licensed behavioral health care staff (52\%) and limited availability of resources and technical assistance to support administrators and staff in developing behavioral health services (43\%). Other than lack of sufficient payment method for behavioral health care services, no other barrier was selected by more than $33 \%$ of BHPs.

Statistically significant differences in response rates existed between PCPs and BHPs for several barriers to integration. PCPs were significantly less likely than BHPs to select lack of community support (OR, $0.3 ; 95 \% \mathrm{CI}, 0.1-0.8)$ as a barrier to integration in their practices and communities. PCPs were significantly more likely than BHPs to 
Table 3. Ideas for Improving Primacy Care and Behavioral Health Integration in the High Plains Research Network

\begin{tabular}{lcccc}
\hline Idea & PCPs $(\mathrm{n}=88)$ & BHPs $(\mathrm{n}=49)$ & Odds Ratio (95\% CI) & $P$ \\
\hline Improve access to BHPs & $50(57)$ & $20(41)$ & $1.9(0.9-3.8)$ & .07 \\
Build an inpatient facility & $30(34)$ & $15(31)$ & $1.2(0.5-2.5)$ & .67 \\
Colocate & $15(17)$ & $25(51)$ & $0.2(0.1-0.4)$ & $0.3(0.1-0.7)$ \\
Warm hand-offs & $33(38)$ & $32(65)$ & $1.2(0.6-2.6)$ & .001 \\
Hire a case manager & $37(42)$ & $18(37)$ & $0.4(0.2-0.9)$ & .54 \\
Shared visits & $19(22)$ & $25(51)$ & $0.4(0.2-0.8)$ & .03 \\
Improve PCP training & $25(28)$ & $23(47)$ & $2.2(1.1-4.5)$ & .03 \\
Improve referral process & $58(66)$ & $2(4)$ & $0.5(0.1-4.0)$ & .62 \\
Nothing & $2(2)$ & &
\end{tabular}

Data are $\mathrm{n}(\%)$ unless otherwise indicated.

$\mathrm{BHP}$, behavioral health provider; CI, confidence interval; PCP, primary care provider.

select lack of sufficient methods of payment for behavioral health care services (OR, 2.7; 95\% CI, 1.3-5.6), recruiting appropriately licensed behavioral health care staff (OR, 3.8; 95\% CI, 1.7-8.3), and retention of appropriately licensed behavioral health care staff $(\mathrm{OR}, 2.7 ; 95 \% \mathrm{CI}, 1.3-5.8)$ as barriers to integration in their practices and communities. PCPs had a slightly but significantly less optimistic response than BHPs regarding the achievability of integration in their communities (Table 5; mean scores, 3.8 and 4.2, respectively; $P<.05$ ).

\section{Discussion}

This study provides new insight into rural provider perspectives about integrated primary care, barriers to integration, and preferred models for improving behavioral health care. Regarding the current status of integration, similar percentages of PCPs and BHPs reported working in a practice that has an integrated BHP. A much larger percentage of BHPs were either considering integrating or in the process of integrating compared with PCPs. These results suggest that $\mathrm{BHPs}$ may more readily adopt integrated practice methods than PCPs, perhaps indicating that the push for integrated primary care often comes from the behavioral health sector.

To improve behavioral health care, PCPs preferred improving the current referral model and were significantly more likely than BHPs to select this option. One possible explanation is that PCPs may feel they are already addressing behavioral health conditions to the fullest extent of their training and an improved referral system is necessary to quickly and efficiently achieve the level of care necessary for more complex patients. Open-ended responses indicated that PCPs desire improved communication with BHPs, especially more timely and thorough referral reports and recommendations. Closed and open-ended responses may be getting at the same root problem: poor communication between the referring provider and the specialist throughout the referral process makes care

Table 4. Barriers to Primary Care and Behavioral Health Integration

\begin{tabular}{|c|c|c|c|c|}
\hline Barrier & $\mathrm{PCP}(\mathrm{n}=88)$ & $\mathrm{BHP}(\mathrm{n}=49)$ & Odds Ratio (95\% CI) & $P$ \\
\hline No champion & $18(20)$ & $12(24)$ & $0.8(0.3-1.8)$ & .58 \\
\hline Lack of payment for BHP & $54(61)$ & $18(37)$ & $2.7(1.3-5.6)$ & $<.01$ \\
\hline Limited resources to develop BHP services & $38(43)$ & $15(31)$ & $1.7(0.8-3.6)$ & .15 \\
\hline No community support & $12(14)$ & $16(33)$ & $0.3(0.1-0.8)$ & $<.01$ \\
\hline PCP recruitment & $19(22)$ & $7(14)$ & $1.6(0.6-4.3)$ & .29 \\
\hline PCP retention & $22(25)$ & $6(12)$ & $2.4(0.9-6.4)$ & .08 \\
\hline BHP recruitment & $46(52)$ & $11(22)$ & $3.8(1.7-8.3)$ & $<.001$ \\
\hline $\mathrm{BHP}$ retention & $46(52)$ & $14(29)$ & $2.7(1.3-5.8)$ & $<.001$ \\
\hline
\end{tabular}

Data are $\mathrm{n}(\%)$ unless otherwise indicated.

$\mathrm{BHP}$, behavioral health provider; $\mathrm{CI}$, confidence interval; PCP, primary care provider. 
Table 5. Level of Agreement That Integration of Primary Care and Behavioral Health in Community is an Achievable Goal

\begin{tabular}{lcc}
\hline Level of Agreement & PCPs $(\mathrm{n}=88)$ & BHPs $(\mathrm{n}=49)$ \\
\hline Strongly disagree & $1(1)$ & $1(2)$ \\
Somewhat disagree & $10(11)$ & $0(0)$ \\
Don't know & $16(18)$ & $9(18)$ \\
Somewhat agree & $32(36)$ & $16(33)$ \\
Strongly agree & $25(28)$ & $21(43)$ \\
Refused & $4(5)$ & $2(4)$ \\
Mean (95\% CI) & $3.8(3.6-4.1)$ & $4.2(3.9-4.5)$ \\
$P$ & & $<.05$ \\
\hline
\end{tabular}

Data are $\mathrm{n}(\%)$ unless otherwise indicated.

BHP, behavioral health provider; CI, confidence interval; PCP, primary care provider.

coordination inefficient. Other open-ended responses hinted that the process for referral to a psychiatrist is a complex multistep process that is difficult and frustrating to navigate for both providers and patients. It is unsurprising that many providers feel the process needs improvement. Open-ended responses by PCPs also suggested that increased staffing and access to BHPs, especially psychiatrists, is necessary.

BHPs were more likely than PCPs to prefer colocation, warm hand-offs, and shared visits. These integrated primary care concepts are found more often in the behavioral health literature than in primary care literature. Therefore, it is likely that BHPs have more exposure to these concepts, are more familiar with the supporting evidence, and are more likely to support efforts to implement these models than PCPs. PCPs may believe that improving the referral process will be a better improvement, but perhaps they are simply less familiar with these concepts or place a higher priority on other aspects of their practice besides behavior health care. In rural communities, PCPs may see complications associated with occasional or visiting BHPs, such as the use of limited examination room space and potential disruption in usual practice routines and patient flow.

BHPs also were more likely than PCPs to prefer improving behavioral health training for PCPs. The notion that behavioral health conditions are inadequately treated in the primary care setting is widely supported by the literature. ${ }^{8-10}$ However, while improving behavioral health education and training for PCPs seems like a logical approach that would improve patient outcomes, these methods have not produced consistent, measurable differences in patient outcomes. ${ }^{12}$ Reviews of depression management found integrated primary care to be more effective than provider educational strategies. ${ }^{13}$

Regarding barriers to integration, it is not surprising that a lack of sufficient methods of payment for behavioral health care services was the barrier most commonly selected by both PCPs and BHPs. There was a marked difference between these 2 groups, however, with a much higher number of PCPs reporting insufficient payment as a barrier. A common concern among HPRN PCPs has been the difficulty in payment for behavioral health services from various payers. Previous studies pointed to a reimbursement system that segregates physical and behavioral health care as a major stumbling block to integration. ${ }^{1,10}$ Open-ended responses echoed this sentiment and indentified specific difficulties with payment, such as lack of reimbursement for behavioral health care provided by PCPs. One provider was unable to hire a licensed clinical social worker because of difficulties in obtaining reimbursement. Despite this being the most commonly selected barrier by both groups, it was selected by only $37 \%$ of BHPs. No other barrier was selected by more than $33 \%$ of BHPs, indicating wide heterogeneity of perspectives regarding integration barriers among this group of professionals. It is unclear why BHPs' perspectives on barriers were so variable and lacked a unifying consensus.

PCPs were significantly more likely than BHPs to select recruitment and retention of appropriately licensed behavioral health care staff as a major barrier. About $87 \%$ of mental health professional shortage areas are in rural areas. This indicates that difficulties recruiting and retaining BHPs disproportionately affect rural areas and are important barriers not just to integration but to access to behavioral health care in general. ${ }^{17}$ Although BHPs were significantly more likely than PCPs to believe that lack of community support was a major barrier to integration, they tended to be slightly more optimistic than PCPs about the achievability of integration.

There were several limitations to this study, including a relatively small and geographically limited sample size. The results are certainly valid for the HPRN and will provide the basis for programs aimed at improving care and integration. We be- 
lieve the results are generalizable to other rural areas in the western United States that have agriculture-based economies and demographics similar to the study population. We intentionally did not include a definition of integrated primary care because there is a lack of consensus on terminology in the field. While this was by design, it does allow for variable interpretations among individuals. Provider input is necessary to put results in context and explain why these discrepancies of opinion between PCPs and BHPs exist. Interviews or focus groups with providers would allow for detailed explanations and be necessary for applying our findings to the actual process of integration in a rural community. This was the first formal research study within the HPRN to include BHPs. While the overall $63 \%$ response is adequate, the small number of BHPs in rural eastern Colorado limit comparisons within this group. Just more than half of behavioral health practices were represented in our data. However, 2 of the respondent practices have multiple sites of care throughout the region. We have identified only 2 psychiatrists who practice in this region, neither of whom live in the region. We are aware of numerous changes (staff, provider, affiliation, etc.) among primary care and behavioral health practices, and our results reflect the findings at the time of the survey.

\section{Conclusion}

This study found that there exist important differences in perspective between rural PCPs and BHPs regarding ideas for improving behavioral health care, barriers to integration, and achievability of integration. These differences may have important implications for rural communities and health care systems that are considering a transition to an integrated primary care model. Solutions may come from the nature of the rural practice itself. In rural communities, the major communication tools are personal relationships and direct communication. Rural PCPs and BHPs may benefit from increased direct communication around patient care, potential for integration, and potential community health programs related to the close connection between physical and behavioral health care. Efforts toward payment reform are making integrated care possible and are moving health care policy toward increased integration. The rural providers in the HPRN reported that integration is an achievable goal. This general support for integrated care, along with policy changes, decreased payment barriers, and improved personal communication between providers may result in increased integration of health care in rural communities.

\section{References}

1. Kathol RG, Butler M, McAlpine DD, Kane RL. Barriers to physical and mental condition integrated service delivery. Psychosom Med 2010;72:511-8.

2. Upshur CC. Crossing the divide: primary care and mental health integration. Adm Policy Ment Health 2005;32:341-55.

3. Zeiss AM, Karlin BE. Integrating mental health and primary care services in the Department of Veterans Affairs health care system. J Clin Psychol Med Settings 2008;15:73-8.

4. Collins C. Integrating behavioral and mental health services into the primary care setting. $\mathrm{N} C \mathrm{Med} \mathrm{J}$. 2009;70:248-52.

5. Regier DA, Goldberg ID, Taube CA. The de facto US mental health services system: a public health perspective. Arch Gen Psychiatry. 1978;35:685-93.

6. Fox J, Merwin E, Blank M. De facto mental health services in the rural south. J Health Care Poor Underserved 1995;6:434-68.

7. McCabe S, Macnee CL. Weaving a new safety net of mental health care in rural America: a model of integrated practice. Issues Ment Health Nurs 2002; 23:263-78.

8. Machado RJ, Tomlinson V. Bridging the gap between primary care and mental health. J Psychosoc Nurs Ment Health Serv 2011;49:24-9; quiz 44.

9. Jameson JP, Blank MB. Diagnosis and treatment of depression and anxiety in rural and nonrural primary care: national survey results. Psychiatr Serv 2010;61: 624-7.

10. Myers K, Stoep AV, Thompson K, Zhou C, Unützer J. Collaborative care for the treatment of Hispanic children diagnosed with attention-deficit hyperactivity disorder. Gen Hosp Psychiatry 2010;32:612-4.

11. Kirchner JE, Cody M, Thrush CR, Sullivan G, Rapp CG. Identifying factors critical to implementation of integrated mental health services in rural VA community-based outpatient clinics. J Behav Health Serv Res 2004;31:13-25.

12. Thielke S, Vannoy S, Unützer J. Integrating mental health and primary care. Prim Care 2007;34:571-92, vii.

13. Gilbody S, Bower P, Fletcher J, Richards D, Sutton AJ. Collaborative care for depression: a cumulative meta-analysis and review of longer-term outcomes. 2006;166:2314-21.

14. Pomerantz AS, Sayers SL. Primary care-mental health integration in healthcare in the Department of Veterans Affairs. 2010;28:78-82. 
15. Bartels SJ, Coakley EH, Zubritsky C, et al. Improving access to geriatric mental health services: a randomized trial comparing treatment engagement with integrated versus enhanced referral care for depression, anxiety, and at-risk alcohol use. Am J Psychiatry 2004;161:1455-62.

16. Farrar S, Kates N, Crustolo AM, Nikolaou L. Integrated model for mental health care. Are health care providers satisfied with it? Can Fam Physician 2001; 47:2483-8.

17. Bird DC, Dempsey P, Hartley D. Addressing mental health workforce needs in underserved rural areas: accomplishments and challenges. Working paper no. 23. Portland (ME): University of Southern Maine,
Edmund S. Muskie School of Public Service, Maine Rural Health Research Center; 2001.

18. Smalley KB, Yancey CT, Warren JC, Naufel K, Ryan R, Pugh JL. Rural mental health and psychological treatment: a review for practitioners. J Clin Psychol 2010;66:479-89.

19. Hester RD. Integrating behavioral health services in rural primary care settings. Subst Abus 2004;25: $63-4$.

20. About HPRN. Aurora (CO): High Plains Research Network; 2014. Available from: http://www. ucdenver.edu/academics/colleges/medicalschool/ departments/familymed/research/PBRN/HPRN/ Pages/AboutHPRN.aspx. Accessed March 1, 2013. 